\title{
Peptide Targeting of an
}

Intracellular Receptor of the Secretory Pathway

\section{Journal Article}

Author(s):

Tirla, Alina; Rivera Fuentes, Pablo (1)

Publication date:

2019-03-05

Permanent link:

https://doi.org/10.3929/ethz-b-000331780

Rights / license:

In Copyright - Non-Commercial Use Permitted

Originally published in:

Biochemistry 58(9), https://doi.org/10.1021/acs.biochem.9b00029

Funding acknowledgement:

165551 - Development of Intracellular Photoactivatable Probes (SNF) 


\title{
Peptide Targeting of an Intracellular Receptor of the Secretory Pathway
}

\author{
Alina Tirla and Pablo Rivera-Fuentes* \\ Laboratorium für Organische Chemie, ETH Zürich, Vladimir-Prelog-Weg 3, 8093 Zürich, Switzerland
}

\begin{abstract}
Numerous peptides serve as natural ligands of intra- and extracellular receptors. The presence of charged amino acids, however, often hinder the membrane-crossing ability of some of these peptides and render them unsuitable as chemical probes to perturb or image intracellular targets. In this report, we show that addition of a few natural and unnatural amino acids enhance the cellular uptake and intracellular localization of a highly charged Lys-Asp-Glu-Leu (KDEL) peptide to target the corresponding receptor of the secretory pathway. Live-cell imaging experiments revealed that, through interaction with the KDEL receptor, the peptide is delivered to the endoplasmic reticulum (ER), where it accumulates preferentially. The enhanced uptake and selectivity of this peptide make it a good probe to monitor disruptions in retrograde transport and ER stress in living cells without any genetic modifications.
\end{abstract}

The secretory pathway is a series of membrane-enclosed organelles that contain the endoplasmic reticulum (ER), the Golgi apparatus, the ER Golgi intermediate compartment (ERGIC), secretory vesicles, and the plasma membrane. ${ }^{1}$ Almost a third of all synthesized proteins are targeted to the ER for folding, assembly, post-translational modifications, and subsequent trafficking to downstream compartments. ${ }^{2}$ These protein modifications are attained by ER-resident proteins, such as folding enzymes and chaperones. ${ }^{3}$ A majority of these chaperones, such as glucose-regulated protein-78 (GRP78), glucose-regulated protein-94 (GRP94), and protein disulfide isomerase (PDI), contain a common C-terminal tetrapeptide motif (Lys-Asp-Glu-Leu, KDEL). The KDEL motif acts as a signal that the KDEL receptor (KDELR) uses to retrieve ER-resident proteins that escaped the ER. ${ }^{4}$

KDELR is a protein receptor that consists of seven trans-membrane domains and is part of the secretory pathway. ${ }^{3}$ It is located either in the cis-Golgi compartment or in the ERGIC. Interaction with the KDEL motif of proteins leads to conformational change and oligomerization of the receptor, which needs to be phosphorylated by protein kinase A to recruit the phosphorylated ADP-ribosylation factor 1 (ARF1-GTP, Figure 1). ${ }^{5,6}$ Next, binding of the coat protein complex I (COPI) induces formation of the acidic COPI vesicles, which are delivered to the ER via retrograde transport. Once in the ER, the nearly neutral $\mathrm{pH}$ of this organelle promotes the dissociation of the cargo-receptor complex and the receptor is recycled.

Apart from its role in maintaining the homeostasis of the secretory pathway, KDELR has additional cellular functions, including activation of Src family kinases ${ }^{7}$, participation in the ER stress response by promoting the phosphorylation of p 38 mitogenactivated protein kinases (MAPKs), ${ }^{3,8}$ and induction of autophagy. ${ }^{9}$ These multiple functions make the KDELR a very interesting target for small-molecule ligands that could reveal basic aspects of cell biology and inspire the development of new therapies.

As a trans-membrane receptor, KDELR shares several structural and functional similarities with $\mathrm{G}$ protein-coupled receptors (GPCRs). ${ }^{3}$ Unlike most GPCRs, however, KDELR is located in intracellular membranes, and therefore targeting this receptor using its highly charged and membrane-impermeant natural peptide ligand, KDEL, has been accomplished only by genetically encoded probes. ${ }^{5,10}$ The availability of a small-molecule probe that could be used not only for binding, but also for imaging, KDELR would provide an invaluable tool to monitor intracellular protein trafficking through the compartments of the secretory pathway.

A successful KDELR probe would have to overcome the poor membrane-crossing ability of the natural peptide ligand, KDEL. We envisioned that additional amino acids, in particular an asparagusic acid-modified lysine (KAspA) could improve the cellular uptake of this short and highly charged peptide (Figure 2A). ${ }^{11,12}$ Additionally, we anticipated that a bright and photostable silicon rhodamine dye would be an efficient fluorescent reporter that would allow us to monitor the uptake and cellular trafficking of KDEL peptide derivatives (synthesis in the Supporting Information, SI). 


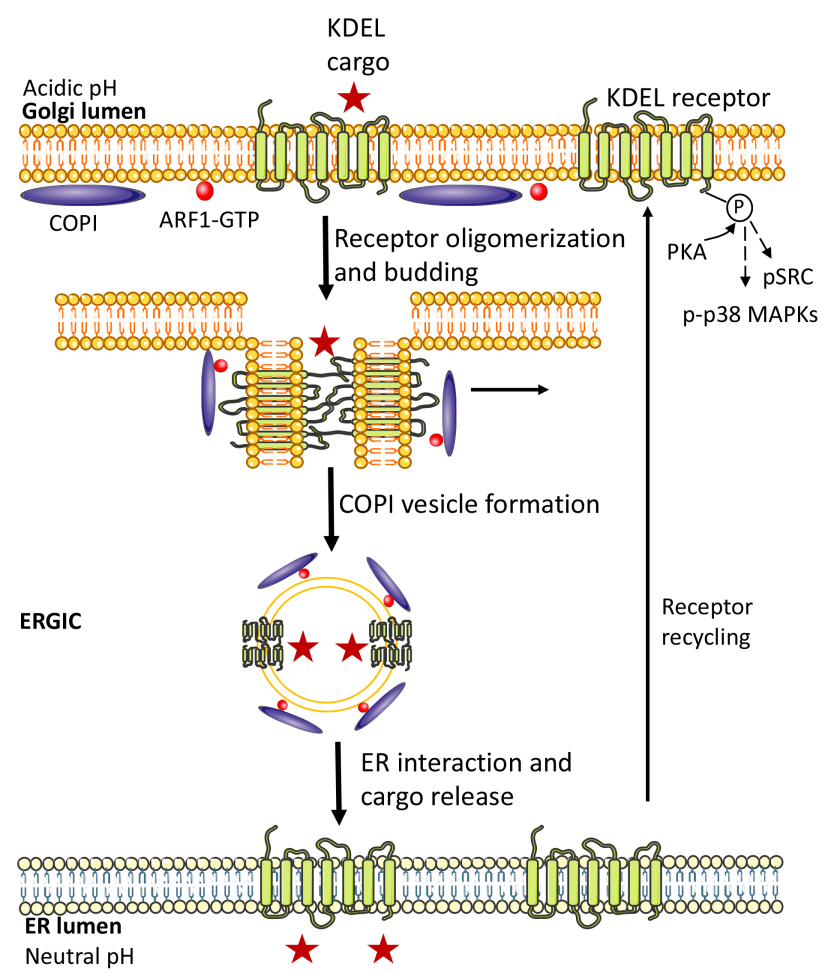

Figure 1. Retrograde transport of KDEL cargo from Golgi to ER. Upon interaction with the KDEL cargo at the cis-Golgi, the phosphorylated KDEL receptor oligomerizes, recruits ARF1-GTP and forms a complex with COPI. This leads to formation of COPI vesicles, which are delivered to the ER. There, the complex dissociates, the cargo is released in the ER and the receptor is recycled back to the Golgi. Activation of the KDELR also has additional effects, such as phosphorylation of Src and of p-38 MAPKs.

In humans, KDELR has three different isoforms that can be targeted preferentially. KDELR1 shows preferential binding to the tetrapeptide -KDEL, KDELR2 to - HXEL ( $\mathrm{X}$ = hydrophobic amino acids) and KDELR3a/b to -HDEL, which is also the preferred sequence for the HDEL receptor, the yeast counterpart of the mammalian KDELR., ${ }^{3,5}$ Using solid-phase peptide synthesis, ${ }^{13}$ we prepared an initial library of three peptides that had C-terminal -KDEL, -HVEL and -HDEL, to represent the preferred binding motifs of the three KDELR isoforms. These peptides were tested for uptake and subcellular distribution by confocal microscopy in live cells. Human cervical cancer cells (HeLa) were incubated with the peptides $(5 \mu \mathrm{M})$ for either 2 or $18 \mathrm{~h}$. In all cases, bright fluorescence was measured in the intracellular medium confirming that the peptides are taken up efficiently by cells.

Successful binding to the KDELR receptor leads to accumulation of the peptide, and its cargo, in the ER. ${ }^{14}$ Therefore, we used ER accumulation as a measure of retrieval of the peptide by KDELR. After $2 \mathrm{~h}$ of incubation, the -HDEL derivative exhibited the best ER retention of the three derivatives, with a Pearson's coefficient with ER Tracker Green ${ }^{\circledR}$ of 0.44 . The other two peptides exhibited a clear preference for acidic vesicles (Figure 2B, C). After $18 \mathrm{~h}$ of incubation, all three peptides accumulated in acidic vesicles. This observation suggests that the peptides are not being retrieved back to the ER efficiently, perhaps as a consequence of weak binding to KDELR in the Golgi and acidic vesicles. 


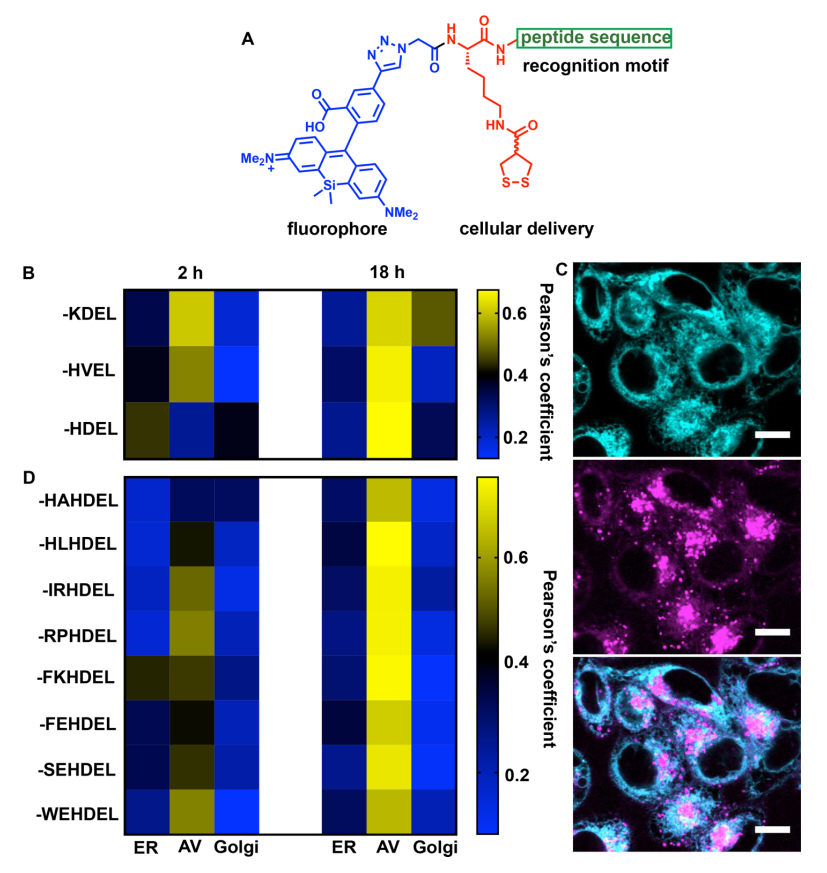

Figure 2. A) Probe design and components; B) Co-localization results of -KDEL, -HVEL and -HDEL peptides, with ER, acidic vesicles (AV) and Golgi apparatus, after 2 and 18 h peptide incubation; C) HeLa cells stained with ER-Tracker Green ${ }^{\circledR}$ (cyan, $1 \mu$ M), HDEL (magenta, $5 \mu \mathrm{M}$ ), and overlay; D) Co-localization results of -XXHDEL peptides with ER, acidic vesicles (AV) and Golgi apparatus, after 2 and $18 \mathrm{~h}$ peptide incubation. Scale bars $=10 \mu \mathrm{m}$.

Because the -HDEL motif seemed to be partially retrieved to the ER at early time points, we used it as lead probe for further development. Using genetically encoded probes, Ruddock and co-workers reported that lengthening the recognition motif to six amino acids can improve interaction of proteins with KDELR. ${ }^{14}$ Specifically, having a positive charge appears to be an important component at position -5 or -6 , and because the residues $-\mathrm{HA}-,-\mathrm{HL}-,-\mathrm{IR}-$ and $-\mathrm{RP}-$ in these positions improved ER retention of proteins, we generated a new library of peptides incorporating these amino acids into our short peptide. Furthermore, lysozyme-SEHDEL was reported to be successfully retained in the ER upon interaction with KDELR, and thus the -SE- dipeptide was also added to the library. ${ }^{15}$ Finally, Mei et al. described that, in the case of yeast, aromatic residues such as $\mathrm{F}$ and W are preferred at the -6 position, with the -WEHDEL sequence exhibiting the best interaction with the HDEL receptor. ${ }^{10}$

Implementing these modifications resulted in a new library of eight peptides, which were assessed for their KDELR retrieval and accumulation in the ER as described before. In these particular cases, lengthening the -HDEL motif did not have the desired effect of increased ER retention, with only -FKHDEL maintaining a moderate Pearson's coefficient of 0.45 after 2 h of incubation, whereas all other peptides were trapped in acidic vesicles. Taking these results into consideration, we decided to test the effect of the same additional amino acids on the KDEL peptide, which displayed partial localization in the Golgi apparatus (Figure 2B). Analysis of the new library identified a hit sequence, -WEKDEL, which had excellent retrieval into the ER with a Pearson's coefficient of 0.69 after $2 \mathrm{~h}$ of incubation (Figure 3A, B). Whereas most of the peptide was found in the ER, some signal can also be seen in acidic vesicles (Figure 3B), which can be expected from dynamic retrograde transport. Furthermore, even after $18 \mathrm{~h}$ incubation, this peptide still displayed good ER localization, indicating that retrieval is efficient and confinement to acidic vesicles and eventual secretion are significantly slowed down compared to other peptides. 


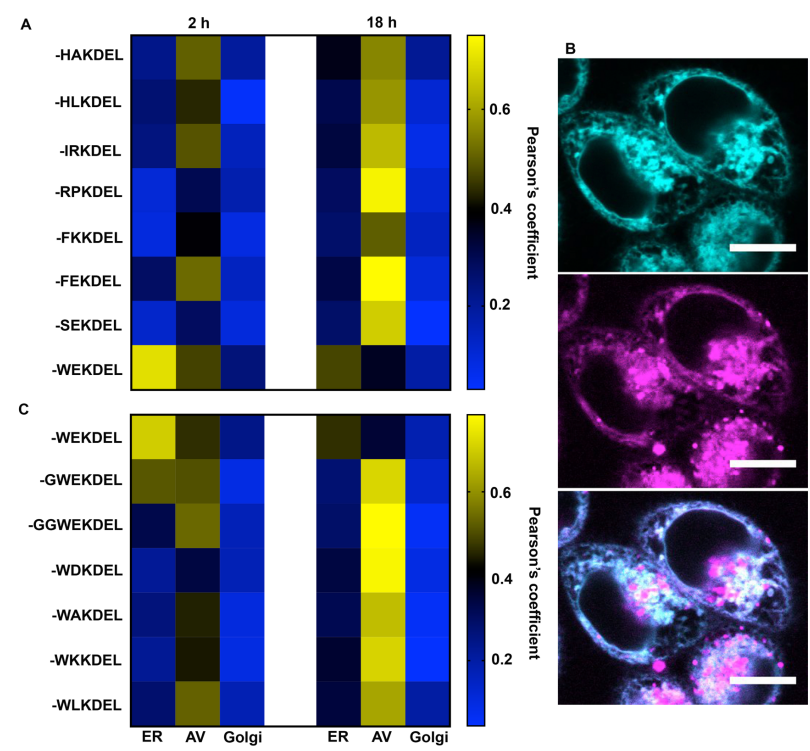

Figure 3. A) Co-localization results of -XXKDEL peptides with ER, acidic vesicles (AV) and Golgi apparatus, after 2 and $18 \mathrm{~h}$ peptide incubation; B) HeLa cells stained with ER-Tracker Green ${ }^{\circledast}$ (cyan, $1 \mu \mathrm{M}$ ), -WEKDEL (magenta, $5 \mu \mathrm{M}$ ), and overlay; C) Colocalization results of -WEKDEL derivatives with ER, acidic vesicles (AV) and Golgi apparatus, after 2 and $18 \mathrm{~h}$ peptide incubation. Scale bars $=10 \mu \mathrm{m}$.

Next, we tested the effect of adding further amino acids between KAspA and -WEKDEL. Addition of a single G residue was tolerated, although it decreased the ER retention of the peptide. Addition of two extra $\mathrm{G}$ residues completely abolished retrieval and the peptide accumulated only in acidic vesicles (Figure 3C). We next examined the role of the amino acid in position -5 , maintaining the $\mathrm{W}$ residue in position -6 . We tested the effect of several types of side chains: negatively charged ( $\mathrm{R}$ ), positively charged (K), small (A), and hydrophobic (L). None of this new peptides displayed retrieval into the ER, confirming the presence of the additional $\mathrm{E}$ residue in position -5 is essential.

Furthermore, we investigated the importance of the asparagusic acid moiety by synthesizing a control peptide in which we replaced the strained cyclic disulfide with a cyclopentane (Figure S2A). Besides exhibiting a substantially lower cellular uptake, colocalization analysis revealed that the cyclopentane derivative had no ER retention and was present mostly in acidic vesicles. This observation could be explained considering that the KAspA amino acid promotes non-endocytic cell entry. ${ }^{[11]}$ This feature prevents sequestration of the peptide in acidic vesicles and allows it to reach the Golgi apparatus, where it can interact with KDELR and enter the secretory pathway (Figure S2B). The enhanced ability of the KAspA derivative to reach the Golgi apparatus might be a consequence of its putative internalization via the transferrin receptor. ${ }^{11}$ This receptor is recycled inside the cell partially through the Golgi compartment, ${ }^{16}$ where the KAspA-labeled peptide could bind KDELR.

KDELR retrieves proteins and peptides from the Golgi apparatus by trapping the cargo in COPI vesicles..$^{5}$ To prove that the hit -WEKDEL peptide is indeed retrieved from the Golgi and delivered to the ER via this mechanism, we prevented the formation of COPI vesicles by dispersing the $\beta$-COP coat from the Golgi lumen using brefeldin A (BFA). ${ }^{17} \mathrm{HeLa}$ cells were pretreated with different concentrations of BFA $\left(10-30 \mu \mathrm{g} \mathrm{mL}^{-1}\right)$ for $1 \mathrm{~h}$, followed by co-incubation of BFA with the -WEKDEL peptide $(5 \mu \mathrm{M})$ for an additional $2 \mathrm{~h}$. Co-incubation is required as the effects of BFA can be reversed over time. ${ }^{17} \mathrm{Co}$ localization analysis of our probe with ER Tracker Green ${ }^{\circledast}$ revealed significant decrease in ER accumulation compared to nontreated cells (Figure 4A). Furthermore, we observed weaker fluorescence intensity of the hit peptide in the ER (Figure S3A), as well as in acidic vesicles (Figure S3B). Because disruption of $\beta$-COP coat should not perturb the cellular uptake of the peptide, this decreased overall fluorescence intensity suggests that by inhibiting retrograde transport, the peptide does not go back to the ER and is instead delivered to secretory vesicles and effluxed. These experiments confirm that the hit -WEKDEL peptide accumulates in the ER via KDELR mediated retrieval and can be used to monitor disruptions in retrograde transport.

The localization and function of KDELR plays a role in the cellular response to ER stress. Upon activation of the unfolded protein response (UPR), the stress response element IRE1 $\alpha$ activates the kinase SRC, which in turn leads to KDELR dispersion from the Golgi and suppression of retrograde transport. ${ }^{8}$ We tested whether hit peptide -WEKDEL could sense suppression of retrograde transport induced by ER stress. Thapsigargin $(\mathrm{Tg})$ is an inhibitor of the ER calcium ATPase that disrupts the calcium 
levels in the ER lumen. ${ }^{18}$ This depletion of calcium abolishes the activity of ER chaperones leading to an increase in protein misfolding and aggregation. To activate the UPR in this way, HeLa cells were pretreated with $\mathrm{Tg}(1-1000 \mathrm{nM})$ for $2 \mathrm{~h}$, followed by co-incubation of $\mathrm{Tg}$ with the -WEKDEL peptide $(5 \mu \mathrm{M})$ for additional $2 \mathrm{~h}$. Co-localization analysis revealed statistically significant decrease in ER retention of the -WEKDEL peptide across all $\mathrm{Tg}$ concentrations tested, indicating that this peptide is indeed capable of detecting UPR-induced KDELR dispersion and suppression of retrograde transport (Figure 4B).
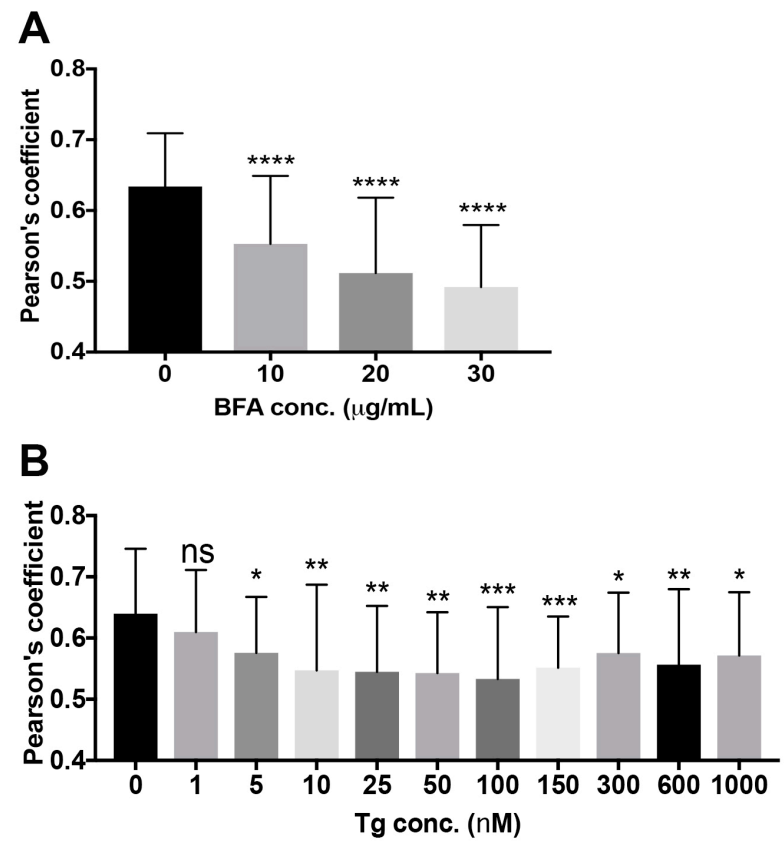

Figure 4. Effect of disrupting the formation of COPI vesicles and UPR induction on cargo retrieval by KDELR A) Co-localization analysis of hit peptide -WEKDEL ( $2 \mathrm{~h}$ incubation) with the ER Tracker Green ${ }^{\circledast}$ in non-treated cells and cells treated with BFA $\left(10-30 \mu \mathrm{g} \mathrm{mL}^{-1}\right)$; B) Colocalization analysis of hit peptide -WEKDEL ( $2 \mathrm{~h}$ incubation) with ER Tracker Green ${ }^{\otimes}$ in non-treated cells and cells treated with the UPR inducer $\operatorname{Tg}(1-1000 \mathrm{nM})$. Measurements were carried out in biological triplicates and data from $n>30$ cells were employed for each condition. Means are plotted and errors bars represent $95 \%$ confidence intervals. In all cases, statistical significance was assessed by unpaired, two-tailed, t-test. $P$ values: ${ }^{* * *}<0.0001$.

In summary, libraries of -HDEL and -KDEL peptides were prepared and tested for targeting KDELR. The hit sequence WEKDEL was efficiently retrieved from the Golgi apparatus and accumulates significantly in the ER, indicating interaction with KDELR. This peptide-based small-molecule probe diffuses into live, unmodified cells and can bring a bright fluorophore to visualize cargo sorting at the ER-Golgi interface. Functionalization with the unnatural amino acid KAspA is essential for efficient uptake and to avoid adventitious accumulation of the peptide in acidic vesicles. Mechanistic biological experiments revealed that the -WEKDEL peptide can be used to study disruptions in retrograde transport by either direct inhibition of coat protein complex formation in COPI vesicles or by activation of the UPR in the ER. Although the KDEL peptide is highly charged and not membrane permeant on its own, we have demonstrated that modification of this sequence with a few natural and unnatural amino acids can lead to a versatile small-molecule probe that can be used to label an intracellular receptor. We envision that this strategy could be used to optimize the cellular uptake and subcellular distribution of ligands for other targets.

\section{ASSOCIATED CONTENT}

Supporting Information.

The Supporting Information is available free of charge on the ACS Publications website.

General methods, cell culture and fluorescence microscopy, co-localization analysis, computational analysis of signal intensity, cell viability assay, synthesis of $\mathrm{SiR}$ and spectroscopic characterization, solid-phase synthesis and peptide characterization, NMR spectra $(\mathrm{PDF})$ 


\section{AUTHOR INFORMATION}

\section{Corresponding Author}

*E-mail: pablo.rivera-fuentes@org.chem.ethz.ch

\section{Funding Sources}

This work was supported by the Swiss National Science Foundation (grant 200021_165551).

\section{Notes}

The authors declare no competing financial interests.

\section{ACKNOWLEDGMENTS}

We thank Mr. Elias A. Halabi (ETH Zürich) for providing the probe for acidic vesicles. We thank the Scientific Center for Optical and Electron Microscopy (ScopeM) at ETH Zürich for access to confocal microscopes.

\section{REFERENCES}

(1) Bonifacino, J. S., and Rojas, R. (2006) Retrograde transport from endosomes to the trans-Golgi network. Nat. Rev. Mol. Cell Biol. $7,568-579$.

(2) Ellgaard, L., and Helenius, A. (2003) Quality control in the endoplasmic reticulum. Nat. Rev. Mol. Cell Biol. 4, 181-191.

(3) Capitani, M., and Sallese, M. (2009) The KDEL receptor: New functions for an old protein. FEBS Lett. 583, 3863-3871.

(4) Stornaiuolo, M., Lotti, L. V., Borgese, N., Torrisi, M.-R., Mottola, G., Martire, G., and Bonatti, S. (2003) KDEL and KKXX retrieval signals appended to the same reporter protein determine different trafficking between endoplasmic reticulum, intermediate compartment, and Golgi complex. Mol. Biol. Cell 14, 889-902.

(5) Raykhel, I., Alanen, H., Salo, K., Jurvansuu, J., Van, D. N., Latva-Ranta, M., and Ruddock, L. (2007) A molecular specificity code for the three mammalian KDEL receptors. J. Cell Biol. 179, 1193-1204.

(6) Majoul, I., Straub, M., Hell, S. W., Duden, R, and Söling, H.-D. (2001) KDEL-Cargo regulates interactions between proteins involved in COPI vesicle traffic: measurements in living cells using FRET. Dev. Cell 1, 139-153.

(7) Giannotta, M., Ruggiero, C., Grossi, M., Cancino, J., Capitani, M., Pulvirenti, T., Consoli, G. M. L., Geraci, C., Fanelli, F., Luini, A., and Sallese, M. (2012) The KDEL receptor couples to Gaq/11 to activate Src kinases and regulate transport through the Golgi. EMBO J. 31, 2869-2881.

(8) Tsai, Y.-L., Ha, D. P., Zhao, H., Carlos, A. J., Wei, S., Pun, T. K., Wu, K., Zandi, E., Kelly, K., and Lee, A. S. (2018) Endoplasmic reticulum stress activates SRC, relocating chaperones to the cell surface where GRP78/CD109 blocks TGF- $\beta$ signaling. Proc. Natl. Acad. Sci. U. S. A. 115, E4245-E4254.

(9) Wang, P., Li, B., Zhou, L., Fei, E., and Wang, G. (2011) The KDEL receptor induces autophagy to promote the clearance of neurodegenerative disease-related proteins. Neuroscience 190, 43-55.

(10) Mei, M., Zhai, C., Li, X., Zhou, Y., Peng, W., Ma, L., Wang, Q., Iverson, B. L., Zhang, G., and Yi, L. (2017) Characterization of aromatic residue-controlled protein retention in the endoplasmic reticulum of Saccharomyces cerevisiae. J. Biol. Chem. 292, 2070720719.

(11) Abegg, D., Gasparini, G., Hoch, D. G., Shuster, A., Bartolami, E., Matile, S., and Adibekian, A. (2017) Strained cyclic disulfides enable cellular uptake by reacting with the transferrin receptor. J. Am. Chem. Soc. 139, 231-238.

(12) Tirla, A., Hansen, M., and Rivera-Fuentes, P. (2018) Synthesis of asparagusic acid modified lysine and its application in solidphase synthesis of peptides with enhanced cellular uptake. Synlett 29, 1289-1292.

(13) Kirin, S. I., Noor, F., and Metzler-Nolte, N. (2007) Manual solid-phase peptide synthesis. J. Chem. Educ. 84, 108-111.

(14) Alanen, H. I., Raykhel, I. B., Luukas, M. J., Salo, K. E. H., and Ruddock, L. W. (2011) Beyond KDEL: The role of positions 5 and 6 in determining ER localization. J. Mol. Biol. 409, 291-297.

(15) Wilson, D. W., Lewis, M. J., and Pelham, H. R. B. (1993) pH-dependent binding of KDEL to its receptor in vitro. J. Biol. Chem. $268,7465-7468$.

(16) Woods, J. W., Doriaux, M., and Farquhar, M. G. (1986) Transferrin receptors recycle to cis and middle as well as trans Golgi cisternae in Ig-secreting myeloma cells. J. Cell Biol. 103, 277-286.

(17) Helms, J. B., and Rothman, J. E. (1992) Inhibition by brefeldin A of a Golgi membrane enzyme that catalyses exchange of guanine nucleotide bound to ARF. Nature 360, 352-354. 
(18) Oslowski, C. M., and Urano, F. (2011) Measuring ER stress and the unfolded protein response using mammalian tissue culture system. Methods Enzym. 490, 71-92. 